\title{
Reflets
}

Revue ontaroise d'intervention sociale et communautaire

\section{Le chemin du retour}

\section{Victoria Monkman}

Volume 3, numéro 2, automne 1997

Visibles et Partenaires : Pratiques et recherches féministes

URI : https://id.erudit.org/iderudit/026186ar

DOI : https://doi.org/10.7202/026186ar

Aller au sommaire du numéro

Éditeur(s)

Reflets : Revue ontaroise d'intervention sociale et communautaire

ISSN

1203-4576 (imprimé)

1712-8498 (numérique)

Découvrir la revue

Citer cet article

Monkman, V. (1997). Le chemin du retour. Reflets, 3(2), 255-259.

https://doi.org/10.7202/026186ar

Tous droits réservés (C) Reflets : Revue ontaroise d'intervention sociale et communautaire, 1997

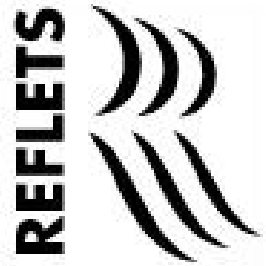

Ce document est protégé par la loi sur le droit d'auteur. L'utilisation des services d'Érudit (y compris la reproduction) est assujettie à sa politique d'utilisation que vous pouvez consulter en ligne.

https://apropos.erudit.org/fr/usagers/politique-dutilisation/ 


\section{Le chemin du retour}

\section{Victoria Monkman}

Intervenante, Studio de la dame bleue, Ottawa

La publication récente du livre Le Chemin du retour: survivre et vivre à la suite de l'abus sexuel ${ }^{1}$ montre que l'action politique et le réseautage peuvent nourrir l'intervention des femmes de l'Ontario français. Ce livre est aussi un outil qui s'adresse à la fois aux survivantes d'agressions à caractère sexuel ou d'abus sexuel et aux intervenantes oeuvrant dans ce domaine.

\section{Un manuel provenant des femmes et des intervenantes}

La nécessité d'écrire un manuel d'accompagnement pour les femmes survivantes d'agressions à caractère sexuel provient des échanges entre les militantes du réseau des Centres d'aide et de lutte contre les agressions à caractère sexuel de l'Ontario (CALACS) et de l'Action ontarienne contre la violence faite aux femmes (AOcVF).

La vision et l'orientation du manuel ont été élaborées à la suite d'échanges avec les membres d'un comité encadreur composé d'intervenantes des programmes francophones dans les centres d'aide et de lutte. Ce comité avait identifié certains principes devant guider la rédaction du manuel d'accompagnement. Pour l'essentiel, ces principes sont les suivants.

- La spécificité franco-ontarienne : les femmes d'expression française ont un statut minoritaire qui les rend vulnérables à l'assimilation. 
- La diversité parmi les Franco-Ontariennes: il existe une grande diversité d'expériences selon les différentes régions, la culture d'origine, l'orientation sexuelle, l'âge, le statut économique ou les habiletés physiques et intellectuelles.

- L'accessibilité du langage : le contenu et le style d'écriture devaient refléter un profond respect des femmes, être ancré dans leur quotidien, tout en prenant en considération le taux élevé d'analphabétisation des Franco-Ontariennes (33\%).

- La perspective féministe: dans un contexte socio-politique, l'agression à caractère sexuel étant perçue comme un abus de pouvoir, l'intervention féministe qui en découle vise la prise de pouvoir personnelle par la survivante et ce par l'entremise d'une intervenante offrant de l'écoute active, du respect et de la confiance dans un processus au rythme de la survivante.

- L'adoption de la perspective d'une survivante s'adressant à d'autres survivantes: afin de s'assurer que cette voix reflète le vécu des survivantes, plusieurs témoignages ont donc été sollicités.

Ce travail de collaboration entre les femmes du comité engagées quotidiennement dans la lutte contre la violence, jumelé aux témoignages de survivantes, a donné lieu à l'élaboration d'un outil dont la communauté francophone avait besoin depuis longtemps.

\section{Aperçu du manuel}

Sans reprendre tout le contenu du manuel, en voici un aperçu. Le tout débute par une définition détaillée des agressions à caractère sexuel et de l'abus sexuel, incluant les abus rituels, les excisions et les infibulations, et traite de leurs effets à court et à long terme. Le chapitre suivant porte sur le cheminement des survivantes et offre des explications sur ce que vivent et ressentent ces femmes lorsqu'elles prennent la décision et les moyens de s'en sortir. Rempli de discussions et d'exercices pratiques sur le dévoilement, la recherche de soutien, la montée des souvenirs, les limites personnelles et la dévictimisation, le livre aborde aussi des sujets angoissants pour les survivantes: gérer les émotions intenses, la 
relation avec son corps, la spiritualité, les relations interpersonnelles, etc. La dernière partie consiste en une analyse des multiples formes d'oppression au fondement de toutes les manifestations de violence :

Tout comme l'abus que nous avons vécu de façon individuelle, l'oppression nous fait vivre les mêmes sentiments de honte, de culpabilité, de peine, de colère, d'impuissance, de perte d'estime de soi, d'anxiété, de vulnérabilité (p. 113).

Le manuel accorde une attention particulière au sexisme et à l'oppression des Franco-Ontariennes. Enfin, les annexes offrent des outils pratiques pour les lectrices (listes de personnes-contacts, activités de détente et de centration sur soi, vérification de la réalité et évaluation des relations).

Ce livre est un outil de conscientisation et d'intervention qui peut servir aux intervenantes qu'elles soient elles-mêmes survivantes ou non. Pour les survivantes, il devient alors un outil de soutien.

\section{Pistes pour l'intervention}

Le plus grand besoin d'une survivante qui chemine vers le mieuxêtre est d'être écoutée, d'avoir enfin un témoin pour dire sa douleur, d'être accompagnée et de ne plus se sentir seule avec sa souffrance. Cela dit, chaque survivante est une personne unique qui doit être accueillie dans sa spécificité et dans la complexité de son interaction avec la dimension sociale. La survivante est la seule à pouvoir définir le rythme et l'intensité de son cheminement. Tenter de définir un processus vers le mieux-être qui serait uniforme à toutes les survivantes est souvent difficile et peut causer du tort à la victime. Par exemple, pour certaines survivantes, la confrontation avec l'abuseur peut se produire immédiatement et peut l'aider à se libérer de sa culpabilité. 
Toutefois, pour d'autres, cette confrontation ne peut pas avoir lieu avant d'avoir évacué une certaine douleur vécue dans le passé. Enfin, certaines choisissent de ne plus jamais avoir de contact avec l'abuseur.

La première tâche d'une intervenante, et de loin la plus importante, est d'écouter la victime, et ce sans jugement, mais avec empathie et respect. L'intervenante doit constamment lutter contre les messages qu'elle a elle-même intériorisés et qui pourraient lui dicter de prendre la survivante en pitié, de minimiser sa situation, de juger ses comportements, de lui donner des conseils ou de vouloir la sauver à tout prix. L'intervenante doit accepter la version de la réalité de la survivante sans tenter de l'altérer, tout en lui offrant un milieu où règnent la sécurité et la confiance. Elle doit pouvoir lui fournir les renseignements nécessaires à son cheminement. Il peut s'agir d'un mot juste, d'une liste de ses droits ou encore de la confirmation de ses propres intuitions.

Malgré la victimisation, la survivante a trouvé les moyens de survivre. Ces moyens ont souvent des conséquences douloureuses comme la toxicomanie ou l'automutilation, mais l'intervenante doit reconnaître que ces moyens sont des mécanismes de défense qui permettent à la survivante de passer à travers une plus grande douleur, soit celle causée par l'abus. Il arrive aussi qu'une personne se présente avec des comportements qui sont le résultat de sa victimisation: boulimie, toxicomanie, dépression, etc. La survivante ne verra pas nécessairement le lien entre ces comportements et les abus qu'elle a pu subir. L'intervenante peut alors l'aider établir à ce lien en posant des questions sur ce qu'elle a vécu pendant son enfance. Il arrive toutefois, qu'une femme ne soit pas prête à faire face à l'agression subie, qu'elle ne s'en souvienne pas ou encore qu'elle ait vécu d'autres formes de traumatismes. Dans ces cas, la patience et la prudence deviennent des alliées pour l'intervenante.

Le chemin vers le mieux-être implique un travail intense aux niveaux physique, émotif, intellectuel et spirituel et ce tant pour la survivante que pour l'intervenante. La survivante s'engage à aller jusqu'au plus profond de son être et n'accorde pas facilement sa confiance. Il est donc important de reconnaître le beau cadeau 
qu'elle offre à l'intervenante en lui demandant de l'accompagner dans son cheminement.

Afin d'être en mesure de continuer à accomplir ce travail à long terme, tout en restant en bonne santé, l'intervenante doit approfondir sa connaissance de soi et rester à l'écoute d'elle-même. Cela lui permet de fixer ses limites. Elle doit aussi apprendre à faire face aux émotions qu'elle ressent et qui sont déclenchées par le cheminement de la survivante. Il peut s'agir d'un sentiment de colère qui viendrait de l'écoute des horreurs racontées par la survivante. Elle peut alors mettre en place ses limites face aux exigences de la survivante qui veut souvent l'inclure dans les scénarios de son passé. Par exemple, en réponse aux demandes d'une survivante, elle peut suggérer une alternative à la manipulation. L'intervenante qui établit ses limites de temps et de disponibilité de façon douce mais ferme et claire, peut offrir un modèle afin que la survivante puisse fixer, à son tour, ses propres limites. Admettre les exigences du travail d'accompagnement permet à l'intervenante d'être attentive à son besoin de détente et d'auto-santé. Bien gérer son stress dans ce type d'intervention n'est pas une extravagance mais une nécessité.

L'agression à caractère sexuel et l'abus sexuel ont amené les survivantes sur un chemin qu'elles n'ont pas choisi, mais grâce au travail pour la survie et pour vivre après de tels abus, les femmes apaisent leurs souffrances. Elles prennent alors un chemin qui leur appartient.

\section{Notes}

1. Les lectrices et lecteurs peuvent se procurer le manuel d'accompagnement Le Chemin du retour: survivre et vivre à la suite de l'abus sexuel, rédigé parVictoria Monkman en 1996, auprès de l'Action ontarienne contre la violence faite aux femmes, 260, rue StPatrick, Ottawa (Ontario) K1N 5K5. Vous pouvez aussi communiquer avec l'organisme en composant le (613) 241-8433 ou par télécopieur au (613) 241-8435. 